\title{
Collective effects in intra-cellular molecular motor transport: coordination, cooperation and competetion *
}

\author{
Debashish Chowdhury ${ }^{1}$ \\ ${ }^{1}$ Department of Physics, Indian Institute of Technology, Kanpur 208016, India.
}

(Dated: July 5, 2021)

\begin{abstract}
Molecular motors do not work in isolation in-vivo. We highlight some of the coordinations, cooperations and competitions that determine the collective properties of molecular motors in eukaryotic cells. In the context of traffic-like movement of motors on a track, we emphasize the importance of single-motor bio-chemical cycle and enzymatic activity on their collective spatio-temporal organisation. Our modelling strategy is based on a synthesis- the same model describes the single-motor mechano-chemistry at sufficiently low densities whereas at higher densities it accounts for the collective flow properties and the density profiles of the motors. We consider two specific examples, namely, traffic of single-headed kinesin motors KIF1A on a microtubule track and ribosome traffic on a messenger RNA track.
\end{abstract}

PACS numbers:

\section{INTRODUCTION}

Molecular motors [1, 2] perform crucial functions at almost every stage in the life of a cell. The motordependent activities begin with DNA replication [3] which is essential before cell division. A motor called DNA helicase [4, 5] unzips the double-stranded DNA so that another motor, called DNA polymerase 6 can synthesize two copies of the DNA using the two single strands as templates. Protein synthesis [3], one of the crucial activities within a cell, is accomplished by some other motors at different stages of operation, the most notable among these are RNA polymerase [7] and ribosomes 8]. Normally, the proteins are synthesized near the cell center and need to be transported to appropriate locations some of which are far from the cell center. Besides, some cargoes are also transported from the cell periphery to the cell center. Most of these transportations of cargoes (often in appropriately packaged form) in the cytoplasm are carried out by yet another set of molecular motors which are collectively referred to as cytoskeletal motors 9 .

The molecular motors at the cellular and subcellular levels can be grouped together is several different ways. One possible way of grouping these is based on the nature of the tracks on which the motors move- one group moves on filamentary proteins whereas the other moves on nucleic acid strands. Among the cytoskeletal motors, which walk on filamentary proteins, kinesin and dynein move on microtubules (MT) whereas myosins move on actin filaments. Some motors are processive, i.e., capable of long walk without getting detached from the track. Kinesins and dyneins are not only processive but can carry

${ }^{*}$ Section $\mathrm{VB}$ is based on original work [59, 67] carried out in collaboration with K. Nishinari, Y. Okada, A. Schadschneider, P. Greulich and A. Garai. Section DI is based on original work 78 done in collaboration with A. Basu. molecular cargo; therefore, these are functionally similar to "porters".

Molecular motors do not work in isolation in-vivo. Wide range of biophysical phenomena observed at subcellular and cellular levels are manifestations of different types of collective processes, involving molecular motors, at several different levels of biological organisation [10]. The nature of the collective effect depends on the situation; it can be the coordination of different parts of a single motor or, on a larger scale, it can be a cooperation or competition between two different motors. These collective effects can manifest as spontaneous oscillations, dynamic instabilities, hysteresis, dynamical phase transitions [11, 12] and motor traffic jam [13]. In this paper, we first give examples of different types of coordination, cooperation and competition in molecular motor transport. Then, we focus on our works on molecular motor traffic on two different types of tracks, namely, on MT and on messenger RNA (mRNA).

\section{COORDINATION THROUGH ELASTIC COUPLING}

In this section we consider coordination of elastically coupled motor domains of a given motor as well as that of motors which are elastically coupled to a common backbone. The structures and mechanisms of single cytoskeletal motors have been discussed in this proceedings by Ray [14], and by Mallik and Gross [15] while those of helicase motors have been reviewed by Tuteja and Tuteja [16].

\section{A. Coordination of different heads of oligomeric motors}

Most of the known common molecular motor proteins are dimeric or, in general, oligomeric [17]. It is generally believed that coordination of the ATPase activities of different motor domains is essential for the processivity 
of a given motor. The mechanism of this coordination has been investiagted extensively over the last decade using many different techniques.

It is now quite well established that the conventional double-headed kinesins follow a hand-over-hand mechanism (exactly similar to the steppings used by humans for walking) 18, 19, 20, 21, 22, 23]. Similar hand-over-hand mechanism is also believed to govern the movement of myosin- $\mathrm{V}$, an unconventional processive myosin 24,25$]$. However, the corresponding mechanism for dimeric cytoplasmic dynein is much more complex because the architecture of its motor domain is very different from those of kinesin and myosin- $\mathrm{V}$ [26, 27].

Majority of the molecular motors that move on nucleic acid tracks (DNA or RNA) are also oligomeric. For example, most DNA helicases are either dimeric or hexameric [4]. Both "inchworm" and "hand-over-hand" (also called "rolling") mechanisms for the coordination of the motor domains of dimeric DNA helicases have been considered 28]. The corresponding mechanism for hexameric DNA helicases remains unclear; at least three different plausible scenarios have been suggested. The ATPase activity of the motor domains in hexameric DNA helicases can run (i) in parallel, or (ii) in ordered sequential, or (iii) in random-sequential manner 29$]$.

\section{B. Collective dynamics of cytoskeletal motors bound to a common elastic backbone}

Consider a group of identical motors bound to an elastic backbone as shown in fig 1 It has been shown [30] that, even if each individual motor is non-processive, such a system of elastically coupled motors can move collectively on a filamentary track in a processive manner in one direction for a period of time and, then, spontaneously reverse its direction of motion. Such spontaneous oscillations can account for the dynamics of axonemes, which are core constituents of eucaryotic cilia, as well as oscillatory motions of flight muscles of many insects [1, 31].

\section{TWO SUPERFAMILIES OF PORTERS: LOAD-SHARING VERSUS TUG-OF-WAR}

Kinesins and dyneins are both processive (i.e., "porters"), but move in opposite directions along MT tracks. Often several members of the same superfamily together carry a single cargo. On the other hand, if members of both the superfamilies are adsorbed simultaneously on the same cargo, they compete against each other trying to pull the cargo in opposite directions. Recent progress in theoretical modelling of these phenomena have been summarized in this proceedings by Lipowsky et al. 32.

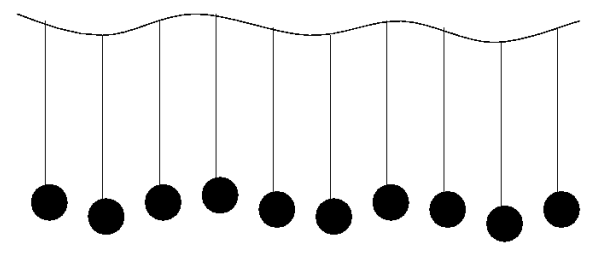

FIG. 1: Schematic description of the model of elastically coupled cytoskeletal motors [30]; the horizontal curve represents the elastic backbone.

\section{A. Unidirectional transport: load-sharing by members of one superfamily}

Two types of in-vitro motility assays have been used for studying cytoskeletal molecular motors. In particular, in the bead assay the filamentary tracks are fixed to a substrate and motors are attached to a micron-sized bead (usually made of glass or plastic). The movement of the bead in the presence of ATP is monitored using appropriate optical micropscopic methods. In such situations, each bead is likely to be covered by $N$ motors where, in general, $N>1$. More than one motor is also used for transportation of large organelles in-vivo. This phenomenon of unidirectional cooperative cargo transport by more than one motor of the same superfamily has been studied theoretically $[33$. The average walking distance increase with increasing $N$. In the face of opposition by external load force, the force is shared by the $N$ motors.

Cooperative pulling of lipid bilayer membranes by kinesin motors can generate membrane tubes [34, 35, [36]. Such minimal systems are adequate to gain insight into the role of motor-mediated interaction between the cytoskeleton and organelles in the formation of endoplasmic reticulum and Golgi membrane networks.

In case of transport by cytoplasmic dynein motors, if more than one motor work together then collectively they can improve the performance as porter although individually, while working in isolation, they often pause and experience slippage in presence of load force [37].

\section{B. Bidirectional transport: tug-of-war between two superfamiles}

In the preceeding section we have mentioned how the a single assembly of elastically coupled motors can sponta- 


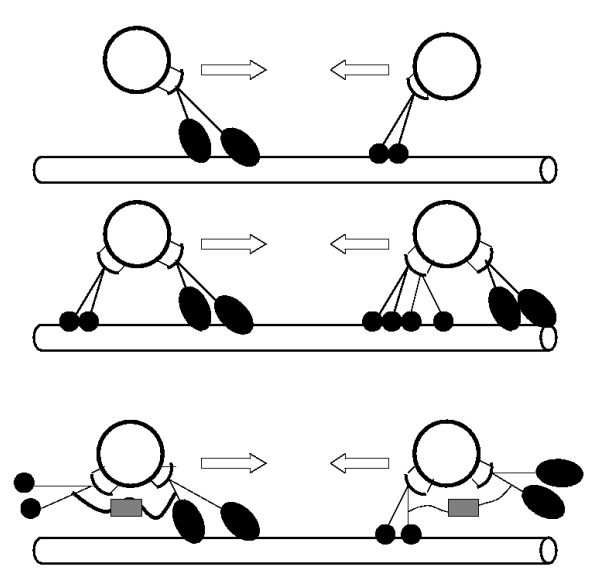

FIG. 2: Schematic description of the three possible mechanisms of bidirectional traffic. The three figures from top to bottom correspond to the three mechanisms (i), (ii) and (iii) explained in the text. The open circle and the rectangular box represent, respectively, a micron-size bead and a regulator of bidirectional transport.

neously reverse its direction of motion on the same track. It is also well known that some motors reverse the direction of motion by switching over from one track to another which are oriented in anti-parallel fashion. In contrast to these types of reversal of direction of motion, we consider in this section those reversals where the cargo uses a "tug-of-war" between kinesins and dyneins to execute bidirectional motion on the same MT track [38, 39]. Several possible functional advantages of bidirectional transport have been conjectured [38, 39].

Wide varieties of bidirectional cargoes have already been identified so far; these include organelles (for example, mitochondria) as well as secretaory vesicles and even viruses. If motion in one direction dominates overwhelmingly over the other, it becomes extremely difficult to identify the movement unambiguously as "bidirectional" because of the limitations of the spatial and temporal resolutions of the existing techniques of imaging.

The main challenge in this context is to understand the mechanisms of this bidirectional transport and those which control the duration of unidirectional movement in between two successive reversals. This insight will also be utilized for therapeutic strategies. For example, the motor or the motor-cargo link may be targeted blocking the virus that hijacks the motor transport system to travel towards the nucleus. On the other hand, a virus executing bidirectional movements can be turned away from the outskirts of the nucleus by tilting the balance in favour of the kinesins.

At least three possible mechanisms of bidirectional transport have been postulated (see fig 21). (i) One possibility is that either only + end directed motors or only - end directed motors are attached to the cargo at any given instant of time. Reversal of the direction of move- ment of the cargo is observed when the attached motors are replaced by motors of opposite polarity. (ii) The second possible mechanism is the closest to the real life "tug-of-war"; the competion between the motors of opposite polarity, which are simultaneously attached to the same cargo and tend to walk on the same filament generates a net displacement in a direction that is decided by the stronger side. (iii) The third mechanism is based on the concept of regulation; although motors of opposite polarity are simultaneously attached to the cargo, only one type of motors are activated at a time for walking on the track. In this mechanism, the reversal of the cargo movement is caused by the regulator when it disengages one type of motor and engages motors of the opposite polarity. For experimentalists, it is a challenge not only to identify the regulator, if such a regulator exists, but also to identify the mechanism used by the regulator to act as a switch for causing the reversal of cargo movement. Dynactin has been identified as a possible candidate for the role of such a regulator 40,41$]$.

\section{TRANSFER FROM MT NETWORKS TO ACTIN NETWORKS: PARK-AND-RIDE TRANSPORT SYSTEM}

The networks of MTs and actin filaments are not disconnected. The cytoskeleton is MT-rich near the cell center whereas dense actin filaments dominate the cytoskeleton near the cell periphery. In recent years functional cooperation between the MT-based and actin-based transport systems have been discovered [42, 43, 44].

Several organisms are known to have an intrinsic ability to manipulate their skin colour. For example, in fish and frog this change of skin colour is caused by the appropriate movement of pigment-containing vesicles called melanosome within a special type of cells called melanophore. When stimulated, melanosomes disperse throughout the cell causing darkening of the skin colour. A second stimulus can reverse the process by aggregation of the melanosomes near the cell center. During dispersion melanosomes are transported, some part of their way towards the cell periphery, by kinesin motors. Then, melanosomes switch from the MT-based kinesin motors to actin-based myosin motor (unconventional myosin$\mathrm{V}$ which is a processive motor) for further distribution around the cell periphery. During the reverse process, the melanosomes begin their journey on the actin-based transportation network and, somewhere along the way, switch over to the MT-based network for continuing their onward journey towards the cell center. This switching of the transport system is similar to park-and-ride systemone drives a car from the suburban areas to the nearest station of the urban high-speed mass transit system (railways or metro trains) for reaching the central part of a city [45].

Cooperation of MT-based and actin-based transportation networks also play crucial roles in neuronal trans- 
port. Actin filaments help in the transport of the cargo to bridge the gaps between MT filaments [42]. The linkers of the two networks and the mechanisms of regulation and control of the proper switchover of cargo from one system to the other have received some attention in the last few years 44, 46, 47].

\section{COLLECTIVE TRANSPORT BY KINESINS: MOLECULAR MOTOR TRAFFIC JAM}

Most of the multi-motor phenomena we have considered in the preceeding section are restricted to sufficiently low densities where direct interaction of the cargoes did not occur. As the cargoes are always much bigger than the motors (in-vitro as well as in-vivo), direct steric interactions of the cargoes become significant when several cargoes are carried by sufficiently dense population of motors along the same track. Such situations are reminiscent of vehicular traffic where mutual hindrance of the vehicles cause traffic jam at sufficiently high densities. In analogy with vehicular traffic, we shall refer to the collective movement of molecular motors along a filamentary track as "molecular motor traffic"; we shall explore the possibility of molecular motor traffic jam and its possible functional implications.

\section{A. TASEP-like minimal models of molecular motor traffic}

Most of the minimal theoretical models of interacting molecular motors 48, 49, 50, 51, 52 utilize the similarities between molecular motor traffic on MT and vehicular traffic on highways [53] both of which can be modelled by appropriate extensions of driven diffusive lattice gases [54, 55]. In such models the motor is represented by a "self-propelled" particle and its dynamics is formulated as an appropriate extension of the totally asymmetric simple exclusion process (TASEP) which is one of the simplest models of interacting driven particles. Many aspects TASEP and its extentions as well as generalizations have been discussed in detail in the articles by Stinchcombe 56], Kolomeisky 57] and Barma 58] in this proceedings. The novel feature of these models, which distinguish these from TASEP are the Langmuir-like kinetics of adsorption and desorption of the motors.

Let us consider the model suggested by Parmeggiani et al. 49]. In this model (see fig 3]) the self-propelled particle can hope from one lattice site to the next immediately in front of it, provided the target site is empty, with hopping probability $q$ per unit time. Moreover, a particle gets attached to an empty site with probability $A$ per unit time time, whereas a particle attached to a site can get detached with probability $D$ per unit time. Furthermore, the attachment probability at the entrance and the detachment probability at the exit are denoted by $\alpha$ and $\beta$, respectively, per unit time. In spite of the extreme sim-

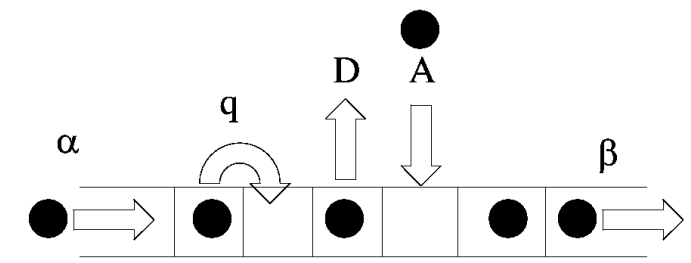

FIG. 3: Schematic description of the model of cytoskeletal motor traffic developed by Parmeggiani et al. 49]

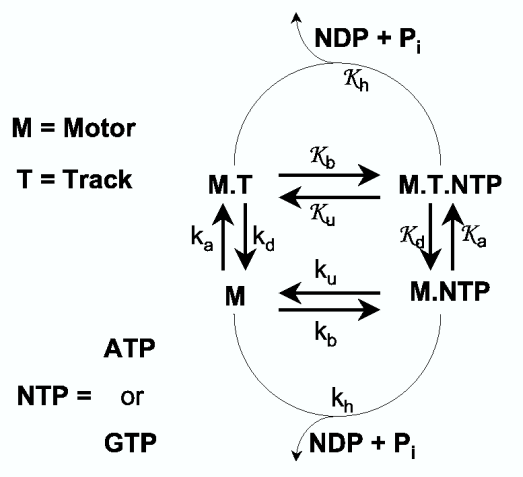

FIG. 4: Biochemical cycle in the absence and presence of the track. The symbols above (or below) the arrows are the rate constants for to the chemical reactions represented by the corresponding arrows.

plicity of this model, it predicted a novel jammed phase on the $\alpha-\beta$ phase diagram. The progress made so far has been reviewed in this proceedings by Lipowsky et al. 32].

\section{B. KIF1A traffic: effects of biochemical cycle}

In reality, a molecular motor is an enzyme that hydrolyses ATP. In the presence of the track, usually the ATPase activity of the motor increases by one or two orders of magnitude; moreover, the mechanical movement of the motor is coupled to its biochemical cycle that includes its enzymatic activity (see fig 4). Models developed for describing the mechanisms of operation of a single motor of a specific type incorporate not only the mechanical states of the motor but also its chemical states in each biochemical cycle. On the other hand, the models of interacting motors in traffic, which we mentioned in the preceeding subsection, do not explicitly take into account the distinct chemical states of each motor during its biochemical cycle. Therefore, one common theme of our recent works on molecular motors has been to achieve a synthesis- we incorporate the essential steps of the biochemical cycle, 
in addition to the mutual interactions of the motors, to develope models for their collective traffic-like dynamics. In the low-density limit, the model describes single-motor dynamics while the same model at higher densities predicts the collective spatio-temporal organization of the motors.

In a recent paper [59] we considered specifically the single-headed kinesin motor, KIF1A [60, 61, 62]; the movement of a single KIF1A motor had already been modelled with a Brownian ratchet mechanism 63, 64]. In contrast to the earlier models 48, 49, 50, 51, 52. of molecular motor traffic, which take into account only the mutual interactions of the motors, our model (from now referred to as the NOSC model) explicitly incorporates also the Brownian ratchet mechanism of individual KIF1A motors, including its biochemical cycle that involves ATP hydrolysis.

The biochemical cycle of a single-headed kinesin motor KIF1A can be described by the four states shown in Fig. [5 60, 62]: bare kinesin (K), kinesin bound with ATP (K.ATP), kinesin bound with the products of hydrolysis, i.e., adenosine diphosphate and phosphate (K.ADP.P), and, finally, kinesin bound with ADP (K.ADP) after releasing phosphate.

Through a series of in-vitro experiments, Okada, Hirokawa and co-workers established that

(i) each KIF1A motor, while weakly bound to a MT, remains tethered to the MT filament by the electrostatic attraction between the positively charged $K$-loop of the motor and the negatively charged $E$-hook of the MT filament.

(ii) In the weakly bound state, a KIF1A cannot wander far away from the MT, but can execute (essentially one-dimensional) diffusive motion parallel to the MT filament. However, in the strongly bound state, the KIF1A motor remains strongly bound to a motor binding site on the MT.

(iii) A transition from the strongly bound state to the weakly bound state is caused by the hydrolysis of the bound ATP molecule. After releasing all the products of hydrolysis (i.e., ADP and phosphate), the motor again binds strongly with the nearest binding site on the MT.

In the NOSC model a single protofilament of MT is modelled by a one-dimensional lattice of $L$ sites each of which corresponds to one KIF1A-binding site on the MT; the lattice spacing is equivalent to $8 \mathrm{~nm}$ which is the separation between the successive binding sites on a MT [9]. Each kinesin is represented by a particle with two possible internal states, labelled by the indices 1 and 2, in which it binds strongly and weakly, respectively, to the MT track. Attachment of a motor to the MT occurs stochastically whenever a binding site on the latter is empty. Such a two-state approximation to the full sequence of biochemical states (conformations) has been successfully exploited also for conventional two-headed kinesin and unconventional myosin- $\mathrm{V}$ both of which are known to be processive [65, 66].

For the dynamical evolution of the system, one of the

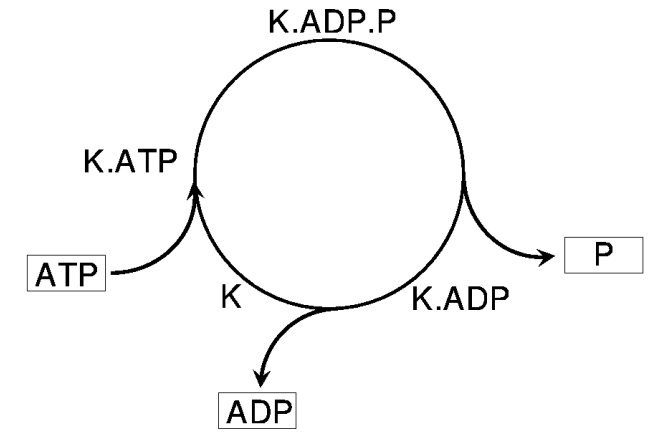

FIG. 5: The biochemical cycle of a single-headed kinesin motor KIF1A.

$L$ sites is picked up randomly and updated according to the rules given below together with the corresponding probabilities (Fig. 6):

$$
\begin{aligned}
& \text { Attachment : } 0 \rightarrow 1 \text { with } \omega_{a} d t \\
& \text { Detachment : } 1 \rightarrow 0 \text { with } \omega_{d} d t \\
& \text { Hydrolysis : } 1 \rightarrow 2 \text { with } \omega_{h} d t \\
& \text { Ratchet: }\left\{\begin{aligned}
2 & \rightarrow 1 \text { with } \omega_{s} d t \\
20 & \rightarrow 01 \text { with } \omega_{f} d t
\end{aligned}\right. \\
& \text { Brownian motion : }\left\{\begin{array}{l}
20 \rightarrow 02 \text { with } \omega_{b} d t \\
02 \rightarrow 20 \text { with } \omega_{b} d t
\end{array}\right.
\end{aligned}
$$

The physical processes captured by the rate constants $w_{f}$ and $w_{s}$ can be understood as follows by analyzing the Brownian ratchet mechanism illustrated in fig [6] For the sake of simplicity, let us imagine that the potential seen by the motor periodically oscillates between the sawtooth shape and the flat shape shown in fig [6] When the sawtooth form remains "on" for some time, the particle settles at the bottom of a well. Then, when the potential is switched "off", the probability distribution of the position of the particle is given by a delta function which, because of free diffusion in the absence of any force, begins to spread. After some time the Gaussian profile spreads to such an extent that it has some overlap also with the well in front, in addition to the overlap it has with the original well. At that stage, when the sawtooth potentil is again switched on, there is a non-vanishing probability that the particle will find itself in the well in front; this probability is proportional to the area of that part of the Gaussian profile which overlaps with the potential well in front and is accounted for in our model by the parameter $w_{f}$. There is also significant probability that the particle will fall back into the original well; this is captured in our model by the parameter $w_{s}$.

Let us denote the probabilities of finding a KIF1A molecule in the states 1 and 2 at the lattice site $i$ at time $t$ by the symbols $r_{i}$ and $h_{i}$, respectively. In mean-field approximation the master equations for the dynamics of 


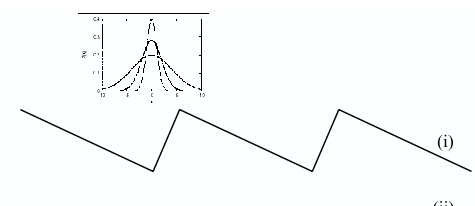

(ii)

FIG. 6: Schematic description of the Brownian-ratchet.

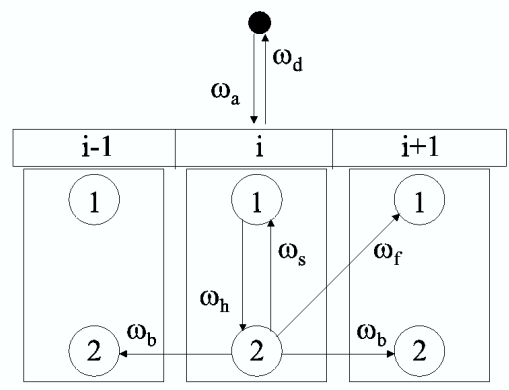

FIG. 7: A schematic representation of the NOSC model of KIF1A traffic. The integer $i$ denotes the discrete equispaced binding sites for kinesin motors on the MT track. The symbols 1 and 2 within circles at a given site $i$ represent the "chemical" (conformational) states of a kinesin at the same spatial location.

motors in the bulk of the system are given by 59

$$
\begin{aligned}
\frac{d r_{i}}{d t}= & \omega_{a}\left(1-r_{i}-h_{i}\right)-\omega_{h} r_{i}-\omega_{d} r_{i}+\omega_{s} h_{i} \\
& +\omega_{f} h_{i-1}\left(1-r_{i}-h_{i}\right), \\
\frac{d h_{i}}{d t}= & -\omega_{s} h_{i}+\omega_{h} r_{i}-\omega_{f} h_{i}\left(1-r_{i+1}-h_{i+1}\right) \\
& -\omega_{b} h_{i}\left(2-r_{i+1}-h_{i+1}-r_{i-1}-h_{i-1}\right) \\
& +\omega_{b}\left(h_{i-1}+h_{i+1}\right)\left(1-r_{i}-h_{i}\right) .
\end{aligned}
$$

The corresponding equations for the boundaries, which depend on the rate constants of entry and exit at the two ends of the MT, are similar and will be presented elsewhere [67].

The single KIF1A properties are reproduced by this model [59] in the appropriate low-density limit. For example, $v$, the mean speed of the kinesins, is about $0.2 \mathrm{~nm} / \mathrm{ms}$ if the supply of ATP is sufficient, and that $v$ decreases with the lowering of ATP concentration following a Michaelis-Menten type relation.
Assuming periodic boundary conditions, the solutions $\left(r_{i}, h_{i}\right)=(r, h)$ of the mean-field equations (7) in the steady-state are found to be

$$
\begin{aligned}
& r=\frac{-\Omega_{h}-\Omega_{s}-\left(\Omega_{s}-1\right) K+\sqrt{D}}{2 K(1+K)}, \\
& h=\frac{\Omega_{h}+\Omega_{s}+\left(\Omega_{s}+1\right) K-\sqrt{D}}{2 K}
\end{aligned}
$$

where $K=\omega_{d} / \omega_{a}, \Omega_{h}=\omega_{h} / \omega_{f}, \Omega_{s}=\omega_{s} / \omega_{f}$, and

$$
D=4 \Omega_{s} K(1+K)+\left(\Omega_{h}+\Omega_{s}+\left(\Omega_{s}-1\right) K\right)^{2} .
$$

The probability of finding an empty binding site on a MT is $K r$ as the stationary solution satisfies the equation $r+h+K r=1$. The corresponding flux is given by 67

$$
\begin{aligned}
J & =W \omega_{f} \\
& =\frac{\omega_{a} \omega_{h} \omega_{f}}{\omega_{f}\left(\omega_{a}+\omega_{d}\right)+\omega_{a}\left(\omega_{s}+\omega_{h}\right)+\omega_{d} \omega_{s}} \\
& =\frac{\omega_{h}(1+K)}{(1+K)\left[(1+K)+\left(\Omega_{s}+\Omega_{h}\right)+\Omega_{s} K\right]} .
\end{aligned}
$$

We have also computed the average density profile of the motors along a MT track with open boundary conditions. For a given $\omega_{a}$, the density of motors in state 2 away from the edges of the MT exceeds that of those in state 1 as $\omega_{h}$ increases beyond a certain value.
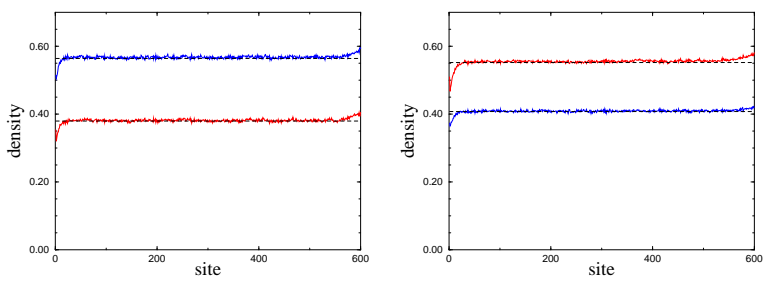

FIG. 8: The stationary density profiles for $\omega_{h}=0.1$ (left) and $\omega_{h}=0.2$ (right) in the case $\omega_{a}=0.001$. The blue and red lines correspond to the densities of state 1 and 2 , respectively. The dashed lines are the mean-field predictions for periodic systems with the same parameters.

In contrast to the phase diagrams in the $\alpha-\beta$-plane reported by earlier investigators [48, 49, 51], we have drawn the phase diagram of our model in the $\omega_{a}-\omega_{h}$ plane by carrying out extensive computer simulations for realistic parameter values of the model with open boundary conditions [59, 67]. The phase diagram shows the strong influence of hydrolysis on the spatial distribution of the motors along the MT. For very low $\omega_{h}$ no kinesins can exist in state 2 ; the kinesins, all of which are in state 1 , are distributed rather homogeneously over the entire system. However, immobile (but fluctuating) shock is observed in the densiti profiles of kinesins if $\omega_{h}$ is finite. 


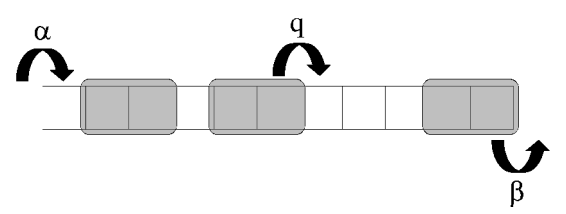

FIG. 9: Schematic description of the traffic of ribosomes where each ribosome has been treated as an "extended" particle $(\ell=2$ in this figure.

\section{RIBOSOME TRAFFIC AND PROTEIN SYNTHESIS}

Translation, the process of synthesis of proteins 68. by decoding genetic information stored in the mRNA, is carried out by ribosomes. Understanding the physical principles underlying the mechanism of operation of this complex macromolecular machine [69] will not only provide insight into the regulation and control of protein synthesis, but may also find therapeutic applications as ribosome is the target of many antibiotics [0].

Most often many ribosomes move simultaneously on the same mRNA strand while each synthesises a protein. In all the earlier models of collective traffic-like movements of ribosomes [71, 72, 73, 74, 75, 76, 77], the entire ribosome is modelled as a single "self-propelled particle" ignoring its molecular composition and architecture. The typical size of an individual ribosome is much larger than that of a single codon (i.e., a triplet of nucleotides). This feature of the ribosome is captured in most of the recent theoretical models by postulating that the size of the selfpropelled particle is $\ell(\ell>1)$ where the unit of length is set by the size of a codon. Moreover, in these models the inter-ribosome interactions are captured through hardcore mutual exclusion and the dynamics of the system is formulated in terms of rules that are essentially straightforward extensions of the TASEP [55]. The qualitative features of the flow properties and spatio-temporal organisation in these models are very similar to those of TASEP which corresponds to the special limit $\ell=1$.

In reality, a ribosome has a complex architecture. Each ribosome consists of two subunits and has four binding sites. Of these, three sites (called E, P, A), which are located in the larger subunit, bind to tRNA, while the fourth binding site, which is located on the smaller subunit, binds to the mRNA strand. The translocation of the smaller subunit of each ribosome on the mRNA track is coupled to the biochemical processes (see fig 10) occuring in the larger subunit.

Let us begin the biochemical cycle with state 1 where a tRNA is bound to the site $\mathrm{P}$ of the ribosome. A tRNAEF-Tu complex now binds to site A and the system makes transition from the state 1 to the state 2 . As long as the $\mathrm{EF}-\mathrm{Tu}$ is attached to the tRNA, codon-antiodon binding

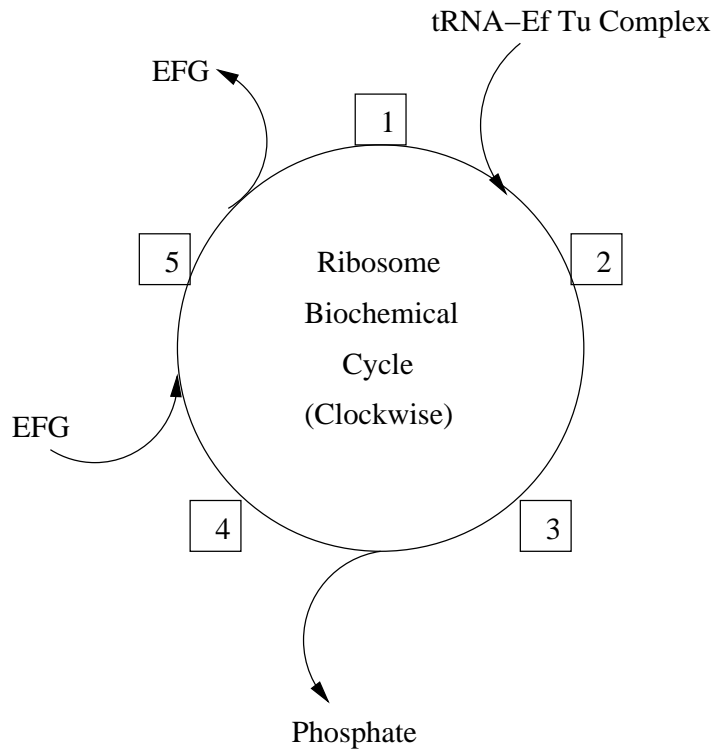

FIG. 10: A simplified biochemical cycle of s ribosome.

can take place, but the peptide bond formation is prevented. The EF-Tu has a GTP part which is hydrolized to GDP, driving the transition from state 2 to 3 . Following this, a phosphate group leaves, resulting in the intermediate state 4. This hydrolysis, finally, releases the EF-Tu, and the peptide formation is now possible. After this step, EF-G, in the GTP bound form, comes in contact with the ribosome. This causes the tRNAs to shift from site $\mathrm{P}$ to $\mathrm{E}$ and from site $\mathrm{A}$ to $\mathrm{P}$, site $\mathrm{A}$ being occupied by the EF-G, resulting in the state 5 . Hydrolysis of the GTP to GDP then releases the EF-G followed by conformatinal changes that are responsible for pulling the mRNA-binding smaller subunit by one step forward. Finally, the tRNA on site A is released, resulting in completion of one biochemical cycle; in the process the ribosome moves forward by one codon [3].

Very recently we [78] have developed a quantitative model that not only incorporates the hard-core mutual exclusion of the interacting ribosomes, but also captures explicitly the essential steps in the biochemical cycle of each ribosome, including GTP (guanine triphosphate) hydrolysis, and couples it to its mechanical movement during protein synthesis. The modelling strategy adopted in ref. 78] for incorporating the biochemical cycle of ribosomes is similar to that followed in our earlier work 59 on single-headed kinesin motors KIF1A. However, the implementation of the strategy is more difficult in case of ribosome traffic not only because of the higher complexity of composition, architecture and mechanochemical processes of the ribosomal machinery but also because of the heterogeneity of the mRNA track 79. The details of our work on ribosome traffic has been reported elsewhere [78]. 


\section{SUMMARY AND CONCLUSION}

In this paper we have presented examples of collective behaviours of molecular motors that emerge from the coordinations, cooperations and competitions at different levels in the sub-cellular world. In our original works so far we have synthesized the single-motor mechanochemistry and multi-motor interactions to develope theoretical models that make experimentally testable quantitative predictions. In particular, we have developed models of KIF1A traffic on a MT track and ribosome traffic on a mRNA track.

Acknowledgements: It is my great pleasure to acknowledge enjoyable collaborations with Aakash Basu, Ashoke Garai, Philip Greulich, Katsuhiro Nishinari, Yasushi Okada and Andreas Schadschneider. I thank Aakash Basu for a critical reading of the manuscript. I also thank Otger Campas, Erwin Frey, Frank Jülicher, Stefan Klumpp, Anatoly Kolomeisky, Reinhard Lipowsky, Gautam Menon and Alex Mogilner for useful discussions over the last few years on various aspects of molecular motors.
[1] M. Schliwa (ed.), Molecular Motors, (Wiley-VCH, 2002).

[2] D. D. Hackney and F. Tamanoi (eds.), The Enzymes, vol.XXIII: energy coupling and molecular motors, (Elsevier 2004).

[3] B. Alberts, A. Johnson, J. Lewis, M. Raff, K. Roberts and P. Walter Molecular Biology of the Cell, (4th edition) (Garland Science, a member of the Taylor and Francis Group, 2002).

[4] M.K. levin and S.S. patel, in ref. [1].

[5] T.M. Lohmann, K. Thorn and R.D. Vale, Cell 93, 9 (1998).

[6] P.J. Rothwell and G. Waksman, in: Adv. in Protein Chem., vol. 71: Fibrous Proteins, eds. J.M. Squire and D.A.D. Parry (Elsevier, 2005).

[7] J. Gelles and R. Landick, Cell 93, 13 (1998).

[8] V. Ramakrishnan, Cell 108, 557 (2002).

[9] J. Howard, Mechanics of motor proteins and the cytoskeleton, (Sinauer Associates, Sunderland, 2001) .

[10] K.C. Vermeulen, G.J.M. Stienen and C.F. Schmidt, J. Muscle res. and cell motility, 23, 71 (2002).

[11] K. Kruse and F. Jülicher, Curr. Op. Cell Biol. 17, 20 (2005).

[12] A. Vilfan and E. Frey, J. Phys. Cond. matt. 17, S3901 (2005).

[13] D. Chowdhury, A. Schsdschneider and K. Nishinari, Phys. of Life Rev. 2, 318 (2005).

[14] K. Ray, in this proceedings.

[15] R. Mallik and S.P. Gross, in this proceedings.

[16] N. Tuteja and R. Tuteja, This proceedings.

[17] G. Mei, A.Di Venere, N. Rosato and A. Finazzi-Agro, FEBS journal, 272, 16 (2005).

[18] R.D. Vale and R.J. Fletterick, Annu. Rev. Cell Dev. Biol. 13, 745 (1997).

[19] D. D. Hackney, in ref.[2].

[20] A. Yildiz and P.R. Selvin, Trends in Cell Biol. 15, 112 (1005).

[21] R. A. Cross, Trends in Biochemical Sciences, 29, 301 (2004).

[22] N.J. Carter and R.A. Cross, Nature, 435, 308 (2005).

[23] N. J. Carter and R. A. Cross, Curr. Op. in Cell Biol. 18, 1 (2006).

[24] R. S. Rock, T.J. Purcell and J.A. Spudich, in: 2]

[25] J. R. Sellers and C. Veigel, Curr. Op. in Cell Biol. 18, 1 (2006).

[26] K. Oiwa and H. Sakakibara, Curr. op. Cell Biol. 17, 98 (2005).

[27] R. Mallik and S.P. Gross, Curr. Biol. 14, R971 (2004).
[28] T.M. Lohmann, J. Hsieh, N.K. Maluf, W. Cheng, A.L. Lucius, C.J. Fischer, K.M. Brendza, S. Korolev and G. Waksman, in ref. [2].

[29] J.C. Liao, Y. J. Jeong, D. E. Kim, S. S. patel and G. Oster, J. Mol. Biol. 350452 (2005).

[30] M. Badoual, F. Jülicher and J. Prost, Proc. natl. Acad. Sci. 99, 6696 (2002).

[31] A. Hilfinger, Ph.D. thesis, Dresden (2005).

[32] R. Lipowsky et al., This proceedings.

[33] S. Klumpp and R. Lipowsky, Proc. Natl. Acad. Sci. USA, 102, 17284 (2005).

[34] A. Roux, G. cappello, J. Cartand, J. Prost, B. Goud and P. Bassereau, Proc. Natl. Acad. Sci. USA 99, 5394 (2002).

[35] G. Koster, M. VanDuijn, B. Hofs and M. Dogterom, Proc. Natl. Acad. Sci. USA 100, 15583 (2003).

[36] C. Leduc, O. Campas, K.B. Zeldovich, A. Roux, P. Jolimaitre, L. Bourel-Bonnet, B. Goud, J.F. Joanny, P. Bassereau and J. Prost, Proc. Natl. Acad. Sci. USA 101, 17096 (2004).

[37] R. Mallik, D. Petrov, S.A. lex, S.J. King and S.P. Gross, 15, 2075 (2005).

[38] M.A. Welte, Curr. Biol. 14, R525 (2004).

[39] S. P. Gross, Phys. Biol. 1, R1 (2004).

[40] S.P. Gross, Curr. Biol. 13, R320 (2003).

[41] K.R. Dell, J. Cell Biol. 160, 291 (2003).

[42] S. S. Brown, in: Annual Rev. Cell Dev. Biol., 15, 63 (1999).

[43] B.L. Goode, D.G. Drubin and G. Barnes, Curr. Op. Cell Biol. 12, 63 (2000).

[44] V. Rodionov, J. Yi, A. Kashina, A. Oladipo and S.P. Gross, Curr. Biol. 13, 1837 (2003).

[45] M. Maniak, Curr. Biol. 13, R917 (2003).

[46] E. Fuchs and Y. Yang, Cell 98, 547 (1999).

[47] E. Fuchs and I. Karakesisoglou, Genes and Development, 15, 1 (2001).

[48] R. Lipowsky, S. Klumpp, and T. M. Nieuwenhuizen, Phys. Rev. Lett. 87, 108101 (2001); R. Lipowsky and S. Klumpp, Physica A 352, 53 (2005) and references therein.

[49] A. Parmeggiani, T. Franosch, and E. Frey, Phys. Rev. Lett. 90, 086601 (2003); Phys. Rev. E 70, 046101 (2004);

[50] E. Frey, A. Parmeggiani and T. Franosch, Genome Informatics, 15(1), 46 (2004).

[51] M.R. Evans, R. Juhasz, and L. Santen, Phys. Rev. E 68, 026117 (2003); R. Juhasz and L. Santen, J. Phys. A 37, 3933 (2004). 
[52] V. Popkov, A. Rakos, R.D. Williams, A.B. Kolomeisky, and G.M. Schütz, Phys. Rev. E 67, 066117 (2003).

[53] D. Chowdhury, L. Santen, and A. Schadschneider, Phys. Rep. 329, 199 (2000).

[54] B. Schmittmann and R.P.K. Zia, in C. Domb and J.L. Lebowitz (eds.), Phase Transitions and Critical Phenomena, Vol. 17 (Academic Press, 1995).

[55] G.M. Schütz, in C. Domb and J.L. Lebowitz (eds.), Phase Transitions and Critical Phenomena, Vol. 19 (Academic Press, 2001).

[56] R. Stinchcombe, This proceedings.

[57] E. Pronina and A.B. Kolomeisky, This proceedings.

[58] M. Barma, This proceedings.

[59] K. Nishinari, Y. Okada, A. schadschneider and D. Chowdhury, Phys. Rev. Lett. 95, 118101 (2005).

[60] Y. Okada and N. Hirokawa, Science 283, 1152 (1999); Proc. Natl. Acad.Sci. USA 97, 640 (2000).

[61] Y. Okada, H. Higuchi, and N. Hirokawa, Nature, 424, 574 (2003).

[62] R. Nitta, M. Kikkawa, Y. Okada, and N. Hirokawa, Science 305, 678 (2004).

[63] F. Jülicher, A. Ajdari, and J. Prost, Rev. Mod. Phys. 69, 1269 (1997).

[64] P. Reimann, Phys. Rep. 361, 57 (2002).

[65] A. B. Kolomeisky and M. E. Fisher, Biophys. J. 84, 1642 (2003).
[66] A. B. Kolomeisky, E.B. Stukalin and A.A. Popov, Phys. Rev. E 71, 031902 (2005).

[67] P. Greulich, A. Garai, K. Nishinari, Y. Okada, A. Schadschneider and D. Chowdhury, to be published.

[68] L.D. Kapp and J. R. Lorsch, Annu. Rev. Biochem. 73, 657 (2004).

[69] A.S. Spirin, FEBS Lett. 514, 2 (2002).

[70] T. Hermann, Curr. Op. in str. biol. 15, 355 (2005).

[71] C.T. MacDonald and J.H. Gibbs, Biopolymers 7, 707 (1969).

[72] G. Lakatos and T. Chou, J. Phys. A 36, 2027 (2003).

[73] L.B. Shaw, R.K.P. Zia and K.H. Lee, Phys. Rev. E 68, 021910 (2003).

[74] L.B. Shaw, J.P. Sethna and K.H. Lee, Phys. Rev. E 70, 021901 (2004).

[75] L.B. Shaw, A.B. Kolomeisky and K.H. Lee, J. Phys. A 37, 2105 (2004).

[76] T. Chou, Biophys. J., 85, 755 (2003).

[77] T. Chou and G. Lakatos, Phys. Rev. Lett. 93, 198101 (2004).

[78] A. Basu and D. Chowdhury, (physics/0604075) and to be published.

[79] Y. Kafri and D.R. Nelson, J. Phys. Cond. Matt. 17, S3871 (2005). 